\title{
RESUMEN
}

La presente investigación analiza la experiencia de divulgación de asuntos claves de auditoría (en adelante, ACA) bajo la norma internacional de auditoría NIA (701) aprobada en Europa el $1^{\circ}$ de enero 2015 por el Consejo Internacional de Normas de Auditoría y Aseguramiento (IAASB). La metodología es descriptiva sobre el contenido de una muestra por conveniencia de 94 ACA en España y 160 ACA en Reino Unido para los períodos 2017 y 2018. El carácter exploratorio se debe a que es un fenómeno poco estudiado en Chile, y es longitudinal porque considera el análisis de información de dos períodos consecutivos. El resultado de esta investigación muestra una tendencia en ambos países de mayor comunicación de ACA en la industria de manufactura. En España por ejemplo, el ACA "Deterioro de Valor" es divulgado mayoritariamente en la industria de manufactura, mientras que en Reino Unido el ACA fue "Provisiones, pasivos contingentes y otras provisiones contables", para las industrias de Retail (primer lugar) y de Transporte (segundo lugar). Dado este estudio, es previsible que los auditores independientes en Chile tenderán a informar los mismos conceptos de riesgos significativos ACA en estructuras de industrias similares y/o iguales, conforme ha sido la experiencia en Europa.

Palabras Claves: asuntos claves de auditoría (ACA), NIA (701), riesgos significativos

\section{NIA 701- EXPERIENCIAS EN ESPAÑA Y REINO UNIDO: DESAFIOS PARA LOS AUDITORES INDEPENDIENTES EN CHILE}

\author{
Marcelo Navarrete Esparza / Juan Carlos Ruiz Cifuentes 2 / Rodrigo Escalona Pradenas ${ }_{3}$
}

\author{
Fechas de recepción: 19 de octubre de 2020 \\ Fecha de aceptación: 10 de noviembre de 2020
}

DOI: https://doi.org/10.22370/riace.2020.9.1.2600

1 Corresponding Author. Departamento de Administración y Auditoría, Facultad de Ciencias Empresariales, Universidad del Bío-Bío, Concepción - Chile, m.navarrete@ubiobio.cl

2 Departamento de Administración y Auditoría, Facultad de Ciencias Económicas y administrativas, Universidad de Concepción, Concepción - Chile, juruiz@udec.cl

3 Departamento de Administración y Auditoría, Facultad de Ciencias Empresariales, Universidad del Bío-Bío, Concepción - Chile, roescalo@alumnos.ubiobio.cl 


\title{
ISA 701 - EXPERIENCES IN SPAIN AND UNITED KINGDOM: CHALLENGES FOR INDEPENDENT AUDITORS IN CHILE
}

\begin{abstract}
This research analyzes the experience of disclosure of key audit issues (hereinafter ACA) under the International Standard on Auditing ISA (701) approved in Europe on January 1, 2015 by the International Auditing and Assurance Standards Board (IAASB). The methodology is descriptive of the content of a sample for convenience of 94 ACA in Spain and 160 ACA in the United Kingdom for the periods 2017 and 2018. The exploratory character is due to the fact that it is a phenomenon that has not been studied much in Chile, and it is longitudinal because it considers the analysis of information from two consecutive periods. The result of this research shows a trend in both countries of greater communication of ACA in the manufacturing industry. In Spain, for example, the ACA "Impairment" is mostly reported in the manufacturing industry, while in the United Kingdom the ACA was "Provisions, contingent liabilities and other accounting provisions", for the Retail (first place) and Transportation industries (second place). Given this study, it is foreseeable that independent auditors in Chile will tend to report the same concepts of significant ACA risks in similar and/or equal industry structures, as has been the experience in Europe.
\end{abstract}

Keywords: key audit issues (KAM), ISA (701), significant risks 


\section{INTRODUCCIÓN}

Actualmente la industria de la auditoría en Chile (cierre períodos 2019 y 2020), no ha implementado los cambios de esta normativa internacional. Los auditores independientes que conforman esta industria en este país se rigen por las Normas de Auditoría Generalmente Aceptadas (NAGA) que se encuentran publicadas por el (Colegio de Contadores de Chile, 2017) y cuyo último compilado de normas fue publicado en el año 2017. La experiencia en Chile en relación a cambios importantes en normas suele tomar bastante tiempo, por ejemplo, el proceso de adopción a las Normas Internacionales de Información Financiera (NIIF) (Silva \& Garrido, 2006), se llevó a cabo el año 2008, no obstante en Europa este proceso de convergencia se implementó en el año 2000. Ad portas que se implemente la NIA (701) este trabajo de investigación permite conocer la experiencia en Europa, y específicamente en industrias similares existentes en Chile. Dada esta situación, se observa una oportunidad para los auditores independientes en Chile en mejorar la revelación actual en sus informes de auditoría, por ello, la presente investigación recoge la experiencia de auditores en Europa para dar respuesta a las siguientes interrogantes; ¿Cuáles fueron los tipos ACA por industrias considerados en $2017 \mathrm{y}$ 2018, en ambos países?, ¿Cuál es la relación entre las variables auditor, empresa y ACA en España y Reino Unido, 2017-2018?, al adoptarse la NIA (701) en Chile, ¿la experiencia sería similar y/o igual a España y Reino Unido?.

Cuatro son las firmas de auditoría (denominadas como las "Big Four": Deloitte, PwC, EY, KPMG) que a nivel mundial lideran el mercado de los servicios de esta industria. De acuerdo a un informe del (International Accounting Bulleting, 2018), en que se publican las cuotas de mercado a nivel de ingresos por ventas en honorarios, la firma Deloitte $(19,4 \%)$ lidera el mercado en el mundo, le sigue PwC $(18,5 \%)$, luego EY $(15,6 \%$ ) y finalmente en el cuarto lugar, KPMG (13\%). El total de ingresos de estas firmas representaron el 66,5\% de las ventas totales a 2018, el otro $33,5 \%$ restante está representado por otras firmas que tienen una cuota de participación considerablemente menor a las de las cuatro firmas de auditoría más representativas de esta industria.

Diversos autores en España (Carreras, Gutiérrez, \& Carmona, 2005; Cuesta \& Garrido, 2015; Ruiz, Rodríguez , \& Biedma, 2015), han escrito para referirse con especial atención al comportamiento de esta industria, el resultado ha sido que existe una alta concentración de este mercado y que presenta características oligopolístico al estar conformados hoy en día por un grupo de cuatro auditoras. Otro aspecto que ha llamado la atención a los investigadores en el tiempo es la baja rotación de los equipos de auditores (Carrera, Humphrey, Gómez, \& Ruiz, 2007), indudablemente esto ha provocado debates internacionales sobre la regulación de la auditoría en este mercado. Con respecto a lo anterior, en Diciembre 2018 el Parlamento Europeo emitió una respuesta dirigida a toda la comunidad europea precisamente refiriéndose a este controversial asunto. La respuesta del parlamento expone sobre posibles distorsiones en las "Big Four" que traen consigo problemas de competencias y conflictos de interés en esta industria. Lo anterior ha suscitado 
preocupación en el Reino Unido, donde el regulador del mercado ha propuesto que las auditorías a las 350 empresas listadas de este país sean auditadas por al menos dos firmas, una de las cuales, no pueda ser "Big Four", con ello este país estaría logrando descomprimir la concentración de mercado.

Otro acontecimiento con efecto colateral en esta industria es la proliferación de escándalos financieros tanto en Europa como en Estados Unidos y en Chile, eventos que surgen como consecuencia de irregularidades, manipulaciones y práctica de contabilidad creativa sobre los estados financieros (Monterrey, 2002), (Morales \& Hollander, 2009), (Cornejo \& Guiñez, 2016), perpetradas por empresas de mucha connotación pública y privada que han traído como consecuencia profundos efectos negativos en inversionistas (minoritarios principalmente), trabajadores y en general stakeholders (Freeman, 1983) los cuales requieren constantemente obtener información para toma de mejores decisiones.

Los acontecimientos ocurridos en la industria de la auditoría a nivel internacional, sumado a otros aspectos de la crisis financiera global iniciada en 2008 activó en Europa una serie de cambios sobre cómo la auditoría podría contribuir a la estabilidad financiera de los mercados, parte de estos cambios se reflejan, por ejemplo, en la creación de la norma internacional de auditoría NIA (701) "Comunicaciones de Asuntos Clave de Auditoría en el informe del auditor Independiente" - Communicating Key Audit Matters in the Independent Auditor's Report (KAM, siglas en inglés), o simplemente denominado "Asuntos Claves de Auditoría" (en adelante, ACA), promulgado por parte del Consejo de Normas Internacionales de Auditoría y Aseguramiento (IAASB, siglas en inglés), y cuya aplicabilidad se sujeta a auditorías que se practican a empresas bajo cotización bursátil.

Inglaterra (Reino Unido) fue el primer país en hacer cambios es sus normas de auditoría (FRC, 2013; Deloitte, 2013; FRC, 2015). Autores, tales como; (Pérez, Camacho, \& Segovia, 2019), reconocen que la investigación previa sobre el impacto de los informes de auditoría ampliados se ha llevado a cabo principalmente en países que conforman el Reino Unido. En España, por su parte y luego de la Reforma de Auditoría de Cuentas (LAC) a nivel de la Unión Europea que entró en vigor el 17 de junio de 2016 para ejercicios que cerraron a partir del 15 de diciembre de 2016, este país inició la emisión de los primeros informes de auditoría de características "ampliados" aplicable a Entidades de Interés Público (EIP), (España, 2015).

En relación a los cambios del IAASB en Europa, y tal como se ha indicado anteriormente, Chile se encuentra atrás respecto a la emisión del nuevo informe ampliado del auditor independiente, por lo tanto se hace necesario llevar a cabo una investigación que analice algunas experiencias de empresas auditadas en España y Reino Unido de tal forma que, tanto auditores como el mercado nacional, hagan previsible los futuros cambios que se deberán encontrar en un período de tiempo no muy lejano sobre la base de ACA informados por auditores "Big Four" 
en los países bajo estudio. Por otra parte, esta investigación ayuda a fortalecer una línea de investigación en auditoría que permita efectuar un seguimiento, antes y a posterior de la implementación de la NIA (701) en Chile, por supuesto, también representa una oportunidad para los auditores en otros países que aún no han efectuado cambios en sus informes.

El presente trabajo se ha basado en el estudio de los autores (Gambetta, García, Sierra, \& Orta, 2019). Dichos autores analizan 144 ACA en distintas empresas cotizadas en el FTSE100 del Reino Unido para los años 2013 y 2016 con el fin de pronosticar si dichos ACA se encontrarán en España una vez que se implemente el nuevo informe "ampliado" en este país a partir del 15 de diciembre de 2016. Este trabajo de investigación fue ampliamente divulgado por el Instituto de Censores Jurados de Cuentas de España (España I. d., 2019).

El objetivo de la investigación es analizar la experiencia a nivel de divulgación de asuntos claves de auditoría ACA que considera 94 ACA en España y 160 ACA en Reino Unido, para los períodos 2017 y 2018. Los ACA informados por los auditores independientes en estos países podrían ser previsible de encontrar en Chile en un futuro no muy lejano. Los autores del presente trabajo consideran que esta información podría ser importante tanto en Chile como para otros países en Latinoamérica que aún no han incorporado los cambios determinados por la NIA (701). La metodología utilizada es descriptiva sobre el contenido de una muestra por conveniencia de 94 ACA en España y 160 ACA en Reino Unido para Ios períodos 2017 y 2018. El carácter exploratorio se debe a que es un fenómeno poco estudiado en Chile, y es longitudinal porque considera el análisis de información de dos períodos consecutivos.

Este trabajo se divide en diferentes secciones. Seguida la introducción se presenta lo siguiente: Se expone el marco teórico, luego, la metodología y datos de la muestra por conveniencia de empresas extraídas de las respectivas bolsas de comercio de cada país (España y Reino Unido) bajo la consideración de industrias más representativas en Chile, y posteriormente se distingue y analiza el contenido de ACA más relevantes informadas por los auditores "Big Four". Finalmente, se presentan los resultados, discusiones, conclusiones y referencias usadas en esta investigación.

\section{MARCO TEÓRICO}

El $1^{\circ}$ de enero de 2015 el IAASB (Consejo de Normas Internacionales de Auditoría y Aseguramiento, 2015, pág. p.51), emite, entre otras normas revisadas y modificadas, la nueva norma internacional de auditoría NIA (701). Esta modificación obligó a los auditores independientes a comunicar en sus informes asuntos claves de auditoria (ACA) dirigidas a la Administración en relación a situaciones que representan riesgos significativos en su examen de auditoría para períodos terminados a partir del 15 de diciembre de 2016, inclusive. Este cambio en el informe de los auditores es 
sustancial y genera oportunidades y como también desafíos para los profesionales en esta industria en Chile. Actualmente, no existe la obligatoriedad de converger a esta norma y los informes actuales de los auditores se basan en la sección AU 700 "Conclusiones de auditoría e Informes" (Colegio de Contador, 2017, pág. 865). La NIA (701) implica mayor involucramiento de juicio profesional por parte del auditor independiente, en cuanto a que deben tomar decisiones para informar a la Administración y encargados del Gobierno en relación a un resumen de los asuntos claves de auditoría de mayor significancia contenidos en su informe. La preocupación del IAASB de aumentar el valor informativo del informe del auditor fue unos de los temas importantes, de manera que el informe de auditoría logre aportar información más amplia al mercado y principalmente a las partes interesadas (internas y externas) (Freeman, 1983). Tanto la Administración como los entes externos (partes interesada) a la empresa pueden generarse ciertas expectativas respecto al resultado final del trabajo de los auditores independientes. Referente a estas expectativas se requiere que los auditores estén alineados, no solo con los requerimientos de las normas de auditoría generalmente aceptadas (por cierto, obligatoria en sus procesos de trabajo), sino también con las expectativas de sus demandantes del servicio (sin que ello comprometa su independencia profesional). Por tanto, generar un sentido práctico con valor agregado de este trabajo para los usuarios, es un reto constante para los auditores independientes.

El autor (Kaplan, 1987) destaca lo siguiente: "La brecha de expectativas entre los auditores y los usuarios de los estados financieros debe cerrarse primero, y la única posibilidad realista de hacerlo requiere que las auditorías reflejen con mayor precisión la comprensión popular de su misión.” (P.1). Lo anteriormente expuesto guarda mucha relación con (Flint, 1988) citado por (Humphrey \& Moizer, 1990), quien sostiene: "La auditoría es un fenómeno social. No tiene ningún propósito 0 valor, excepto en su utilidad práctica (...). La función ha evolucionado en respuesta a una necesidad percibida de individuos o grupos en la sociedad que buscan información o tranquilidad sobre la conducta o el desempeño de otros en los que tienen un interés reconocido o legítimo" (P.1).

La emisión e implementación de la NIA (701) ha venido en cierta forma a acortar la brecha de expectativas entre la Administración y sus auditores independientes responsabilizando a estos últimos de la debida diligencia en comunicar asuntos claves de auditoria (ACA) en su informe, información que hasta antes de esta norma se encontraba sólo en los papeles de trabajo de los auditores. Lo anteriormente expuesto implica que el auditor ejerza el debido juicio profesional para separar y seleccionar aquellos ACA que representaron riesgos más significativos durante todo el proceso de su examen a los estados financieros, indudablemente esto requiere de profesionales experimentados en escepticismo profesional y bien entrenados capaz de asumir una función de auditoría activa, desde la etapa de la planificación hasta la aplicabilidad de los procedimientos sustantivos y cierre de sus papeles de trabajo, incluso, a posterior de haber emitido su informe del auditor independiente a la Administración. Con respecto al informe del auditor el autor (Robalino, 2017), señala: "El informe del auditor independiente es el documento mediante el cual, 
el auditor comunica los resultados obtenidos en el proceso de auditoría, teniendo como finalidad proporcionar información más relevante a los inversores y a los usuarios de información financiera" (P.17).

\section{METODOLOGIA DE LA INVESTIGACIÓN}

En primer lugar incluye un estudio de las industrias representativas en Chile. Una vez seleccionadas las industrias, posteriormente las mismas fueron localizadas en los mercados de España y Reino Unido períodos 2017 y 2018. La fuente de información utilizada es el informe de resultados de Empresas en Chile "Cuarta encuesta longitudinal de Empresa" publicado por el Gobierno de Chile a través del Ministerio de Economía Fomento y Turismo (Chile G. d., 2017). En segundo lugar, se llevó a cabo el levantamiento de la muestra por conveniencia de los 94 ACA en España y 160 ACA en Reino Unido, información que se extrae de los informes de los auditores independientes de empresas listadas en las bolsas de comercio de cada país y que provienen de las páginas web (IBEX en España y FTSE en Reino Unido). Tabla 1 muestra el número de empresas consideradas que listan en bolsas de comercio de cada país.

Tabla 1. Muestra Seleccionada.

\begin{tabular}{lccccc}
\hline \multicolumn{5}{c}{ Estructura de la muestra No de Empresa / ACA } \\
\hline País & $\mathrm{N}^{\circ}$ empresas & IBEX 35 & No - IBEX 35 & $\begin{array}{c}\mathrm{N}^{\circ} \\
\text { ACA }\end{array}$ \\
\hline España & 35 & 7 & 28 & 94 \\
\hline & & & & & \\
\hline País & $\mathrm{N}^{\circ}$ empresas & FTSE 100 & FTSE 250 & $\begin{array}{c}\text { FTSE } \\
\text { ASI }\end{array}$ & $\begin{array}{c}\mathrm{N}^{\circ} \\
\text { ACA }\end{array}$ \\
\hline Reino Unido & 45 & 19 & 21 & 5 & 160 \\
\hline
\end{tabular}

Fuente: Elaboración propia a partir de la muestra por conveniencia

Para lograr el propósito de esta investigación y responder a las interrogantes generadas se trabaja con el levantamiento de la muestra y se analiza de manera descriptiva el contenido individual de ACA revelado en informes emitidos por auditores independientes correspondiente a las "Big Four" y cuyos ACA representan riesgos significativos acerca de información financiera emitidos en estos países. Tanto la estructura del informe del auditor independiente como los ACA analizados se realizan a luz de la NIA (701) aprobada el $1^{\circ}$ de enero 2015 por el IAASB. Con el objeto de profundizar este estudio descriptivo/exploratorio, se aplicó estadística descriptiva univariada y bivariada y análisis de correspondencia múltiple (ACM) de manera de buscar relaciones a nivel de auditores - industria - período, cuya información se presentan en tablas obtenidos del software SPSS v.18. 


\section{ANÁLISIS DE LOS RESULTADOS}

\section{Análisis de los tipos de ACA por industria en España 2017-2018}

Tabla 2 muestra que la actividad de manufactura presenta la mayor cantidad (45) de ACA $(47,87 \%$ y y los ACA mayormente presente en los informes de los auditores corresponde a "Deterioro de Valor" (26), los cuales son predominantes en la industria de manufactura con 15 de 45 ACA. Tabla 3 ratifica que la industria de manufactura informa la mayor cantidad (43) de ACA (45,74\%), del mismo modo el ACA mayormente informado es "Deterioro de Valor" (22) que sitúa a la industria de manufactura con mayor ACA 14 de 43.

Tabla 2. Tipos de ACA por industrias - España 2017.

\section{Cantidad de ACA por industria}

Tipo de ACA $\begin{aligned} & \text { Actividades } \\ & \text { de Servicios }\end{aligned}$ Construcción Manufactura Retail Transporte Total

\begin{tabular}{|c|c|c|c|c|c|c|}
\hline $\begin{array}{l}\text { Valorización } \\
\text { activos }\end{array}$ & 6 & 2 & 3 & 1 & 0 & 12 \\
\hline $\begin{array}{l}\text { Reconocimiento } \\
\text { ingresos }\end{array}$ & 2 & 5 & 6 & 1 & 2 & 16 \\
\hline $\begin{array}{l}\text { Activos por } \\
\text { impuestos } \\
\text { diferidos }\end{array}$ & 3 & 3 & 9 & 2 & 0 & 17 \\
\hline Deterioro de valor & 0 & 5 & 15 & 4 & 2 & 26 \\
\hline $\begin{array}{l}\text { Provisiones, } \\
\text { pasivos } \\
\text { contingentes y } \\
\text { otras provisiones } \\
\text { contables }\end{array}$ & 0 & 3 & 3 & 0 & 4 & 10 \\
\hline $\begin{array}{l}\text { Combinación de } \\
\text { negocios }\end{array}$ & 0 & 0 & 3 & 1 & 0 & 4 \\
\hline Otros & 1 & 1 & 6 & 1 & 0 & 9 \\
\hline Total & 12 & 19 & 45 & 10 & 8 & 94 \\
\hline
\end{tabular}

Fuente: Elaboración propia a partir de la muestra por conveniencia 
Tabla 3. Tipos de ACA por industrias - España 2018

Cantidad de ACA por industria

\begin{tabular}{|c|c|c|c|c|c|c|}
\hline Tipo de ACA & $\begin{array}{l}\text { Actividades } \\
\text { de Servicios }\end{array}$ & Construcción & Manufactura & Retail & Transporte & Total \\
\hline $\begin{array}{l}\text { Valorización } \\
\text { activos }\end{array}$ & 6 & 3 & 8 & 2 & 1 & 20 \\
\hline $\begin{array}{l}\text { Reconocimiento } \\
\text { ingresos }\end{array}$ & 2 & 5 & 4 & 1 & 2 & 14 \\
\hline $\begin{array}{l}\text { Activos por } \\
\text { impuestos } \\
\text { diferidos }\end{array}$ & 3 & 3 & 10 & 2 & 0 & 18 \\
\hline Deterioro de valor & 0 & 3 & 14 & 4 & 1 & 22 \\
\hline $\begin{array}{l}\text { Provisiones, } \\
\text { pasivos } \\
\text { contingentes y } \\
\text { otras provisiones } \\
\text { contables }\end{array}$ & 0 & 2 & 2 & 1 & 4 & 9 \\
\hline $\begin{array}{l}\text { Combinación de } \\
\text { negocios }\end{array}$ & 0 & 0 & 0 & 1 & 0 & 1 \\
\hline Otros & 1 & 0 & 5 & 3 & 1 & 10 \\
\hline Total & 12 & 16 & 43 & 14 & 9 & 94 \\
\hline
\end{tabular}

Fuente: Elaboración propia a partir de la muestra por conveniencia

\section{Análisis de los tipos de ACA por industrias en Reino Unido 2017-2018}

Tabla 4. Presenta la mayor cantidad de ACA (66) está en la industria manufactura $(40,74 \%)$, no obstante el ACA mayormente informado es "Provisiones, pasivos contingentes y otras provisiones contables" (47) que mayoritariamente está en la industria de Retail con 17 de 45. Tabla 5. Presenta la mayor cantidad de ACA (59) en la industria manufactura (36,87\%), y al igual que en 2017, el ACA mayormente informado es "Provisiones, pasivos contingentes y otras provisiones contables" (43) presente de manera importante en la industria de Retail con 16 de 52. 
Tabla 4. Tipos de ACA por industrias Reino Unido 2017

\begin{tabular}{|c|c|c|c|c|c|c|}
\hline & \multicolumn{5}{|c|}{ Cantidad de ACA por industria } & \multirow{2}{*}{ Total } \\
\hline Tipo de ACA & $\begin{array}{l}\text { Actividades } \\
\text { de Servicios }\end{array}$ & Construcción & Manufactura & Retail & Transporte & \\
\hline Valorización activos & 3 & 6 & 5 & 0 & 0 & 14 \\
\hline Reconocimiento de ingresos & 3 & 2 & 11 & 5 & 4 & 25 \\
\hline Deterioro de valor & 1 & 0 & 10 & 10 & 4 & 25 \\
\hline $\begin{array}{l}\text { Provisiones, pasivos } \\
\text { contingentes y otras } \\
\text { provisiones contables }\end{array}$ & 2 & 3 & 13 & 17 & 12 & 47 \\
\hline Combinación de negocios & 1 & 0 & 5 & 2 & 0 & 8 \\
\hline Otros & 1 & 0 & 7 & 3 & 0 & 11 \\
\hline Activos intangibles & 0 & 0 & 1 & 1 & 6 & 8 \\
\hline $\begin{array}{l}\text { Activos por impuestos } \\
\text { diferidos, impuestos fiscales } \\
\text { y precios de transferencia }\end{array}$ & 0 & 0 & 12 & 1 & 1 & 14 \\
\hline Instrumentos financieros & 0 & 0 & 1 & 1 & 2 & 4 \\
\hline $\begin{array}{l}\text { Presentación y revelación de } \\
\text { partidas excepcionales y/o no } \\
\text { subyacentes }\end{array}$ & 0 & 0 & 1 & 5 & 0 & 6 \\
\hline Total & 11 & 11 & 66 & 45 & 29 & 162 \\
\hline
\end{tabular}

Fuente: Elaboración propia a partir de la muestra por conveniencia 
Tabla 5. Tipos de ACA por industrias Reino Unido 2018.

\begin{tabular}{|c|c|c|c|c|c|c|}
\hline \multirow[b]{2}{*}{ Tipo de ACA } & \multicolumn{6}{|c|}{ Cantidad de ACA por industria } \\
\hline & $\begin{array}{l}\text { Actividades } \\
\text { de Servicios }\end{array}$ & Construcción & Manufactura & Retail & Transporte & Total \\
\hline Valorización activos & 3 & 6 & 2 & 1 & 0 & 12 \\
\hline $\begin{array}{l}\text { Reconocimiento de } \\
\text { ingresos }\end{array}$ & 2 & 1 & 12 & 4 & 4 & 23 \\
\hline Deterioro de valor & 1 & 0 & 11 & 10 & 3 & 25 \\
\hline $\begin{array}{l}\text { Provisiones, pasivos } \\
\text { contingentes y otras } \\
\text { provisiones contables }\end{array}$ & 2 & 4 & 9 & 16 & 12 & 43 \\
\hline Combinación de negocios & 0 & 0 & 5 & 2 & 0 & 7 \\
\hline Otros & 0 & 0 & 6 & 3 & 1 & 10 \\
\hline Activos intangibles & 0 & 0 & 1 & 0 & 4 & 5 \\
\hline $\begin{array}{l}\text { Activos por impuestos } \\
\text { diferidos, impuestos } \\
\text { fiscales y precios de } \\
\text { transferencia }\end{array}$ & 0 & 0 & 10 & 2 & 1 & 13 \\
\hline Instrumentos financieros & 0 & 0 & 1 & 1 & 2 & 4 \\
\hline $\begin{array}{l}\text { Presentación y revelación } \\
\text { de partidas excepcionales } \\
\text { y/o no subyacentes }\end{array}$ & 0 & 0 & 1 & 5 & 2 & 8 \\
\hline $\begin{array}{l}\text { NIIF } 16 \text { "arrendamientos" } \\
\text { Efectos o impactos por } \\
\text { implementación }\end{array}$ & 0 & 0 & 1 & 6 & 0 & 7 \\
\hline Brexit & 0 & 1 & 0 & 2 & 0 & 3 \\
\hline Total & 8 & 12 & 59 & 52 & 29 & 160 \\
\hline
\end{tabular}

Fuente: Elaboración propia a partir de la muestra por conveniencia

\section{Análisis de correspondencia múltiple dos dimensiones - España 2017 y 2018}

El siguiente análisis contempla 3 factores en estudio para lo cual se realizó Análisis de Correspondencias Múltiple (ACM) conforme lo establecen los autores (Lizasoain \& Joaristi, 2012) para cada país y en cada año, con el objeto de observar y analizar las principales asociaciones entre las categorías de las variables tipo de industria, auditor y tipo de ACA informado, además, comparar si las asociaciones en cada país de un año a otro se mantienen o sufren variación.

Gráfico 1 y Gráfico 2, analizan y comparan los resultados de ambos años. Al respecto, se observa que las semejanzas y/o relaciones que se mantuvieron en el 2018, por ejemplo; La relación entre la industria de transporte a recibir informes del ACA "provisiones, pasivos contingentes y otras provisiones contables" principalmente por la firma auditora EY. Por otra parte, la relación entre la industria de actividades de servicios a recibir informes del ACA "valorización activos" por el auditor Deloitte. Del mismo modo, la semejanza o similitud entre industrias de retail y manufactura, indica que poseen casi las mismas firmas auditoras 0 que fueron auditadas por casi las mismas firmas y, fueron informadas por casi los 
mismos tipos de ACA. Se observa también la relación del auditor Deloitte a una mayor frecuencia de ACA informados a la industria de actividades de servicios y a construcción, además, la relación de la industria de construcción a recibir informes de ACA "reconocimiento ingresos". Finalmente, se observa relación entre la industria de manufactura en recibir informes de ACA "otros", por PWC, y la relación de manufactura en recibir informes de los ACA "otros" y "deterioro de valor".

Gráfico 1: ACM de dos dimensiones, España 2017

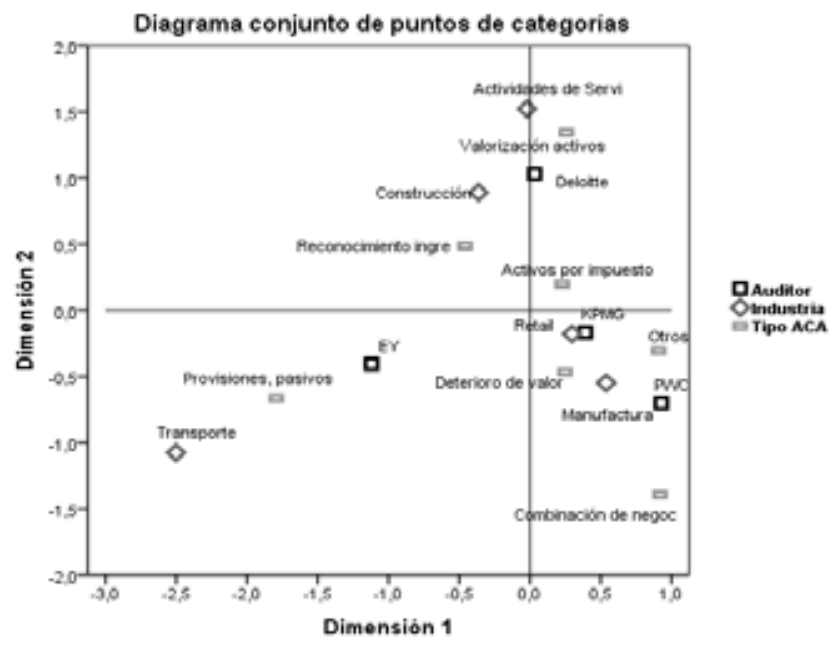

Normalización principal por variable.

Fuente: Elaboración propia

Gráfico 2: ACM de dos dimensiones, España 2018

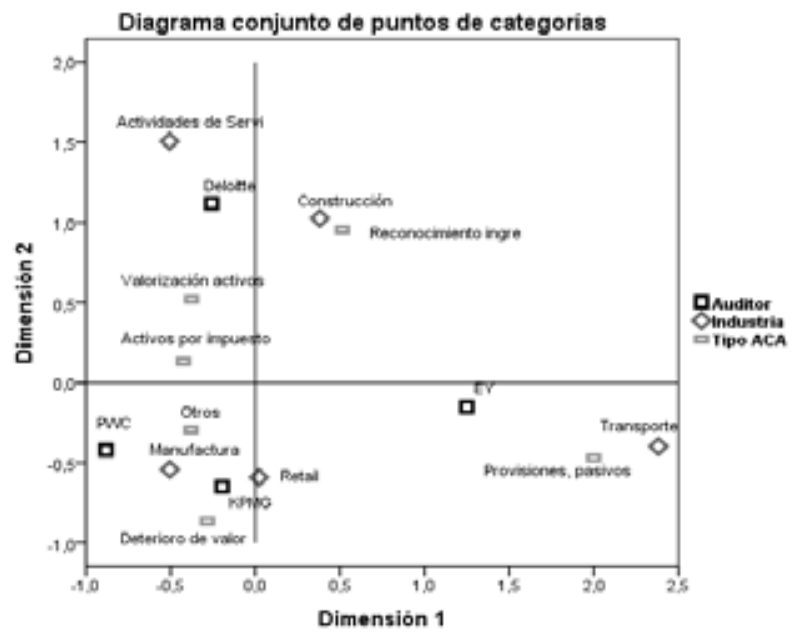

Normalzación principal por variable.

Fuente: Elaboración propia 
Por otra parte, las diferencias o cambios en algunas relaciones de un año a otro, son; La relación que se observó en 2017 del auditor KPMG en informar mayormente Ios ACA "deterioro de valor" y "activos por impuesto diferidos", cambia en 2018, solamente se observó una relación de KPMG en informar el ACA "deterioro de valor". Otra diferencia detectada es la relación en 2017 de la industria de retail en recibir informes de ACA "deterioro de valor" y "activos por impuesto diferidos", cambia en 2018, y sólo se observó que retail está relacionado en recibir informes del ACA "deterioro de valor" (al igual que manufactura), se puede concluir que éste ACA está relacionado en ser informado a la industria de retail y de manufactura. Por último, la relación del ACA "combinación de negocios" en ser informado a manufactura por el PWC en 2017, solo quedó en un informe a la industria de retail por el auditor KPMG el año 2018.

Gráfico 3: ACM de dos dimensiones, Reino Unido 2017.

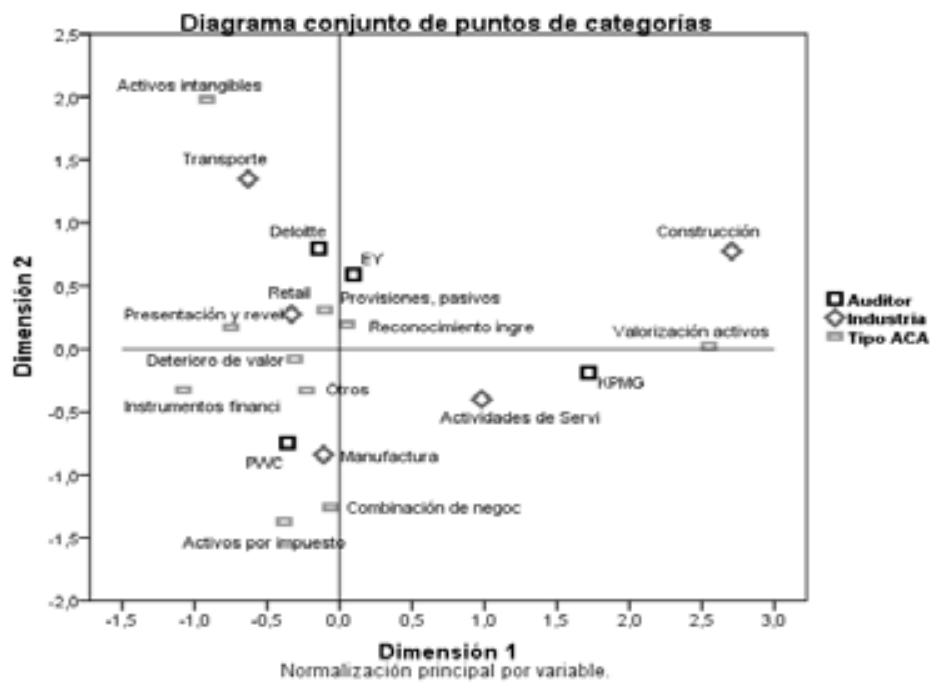

Fuente: Elaboración propia 
Gráfico 4: ACM de dos dimensiones, Reino Unido 2018.

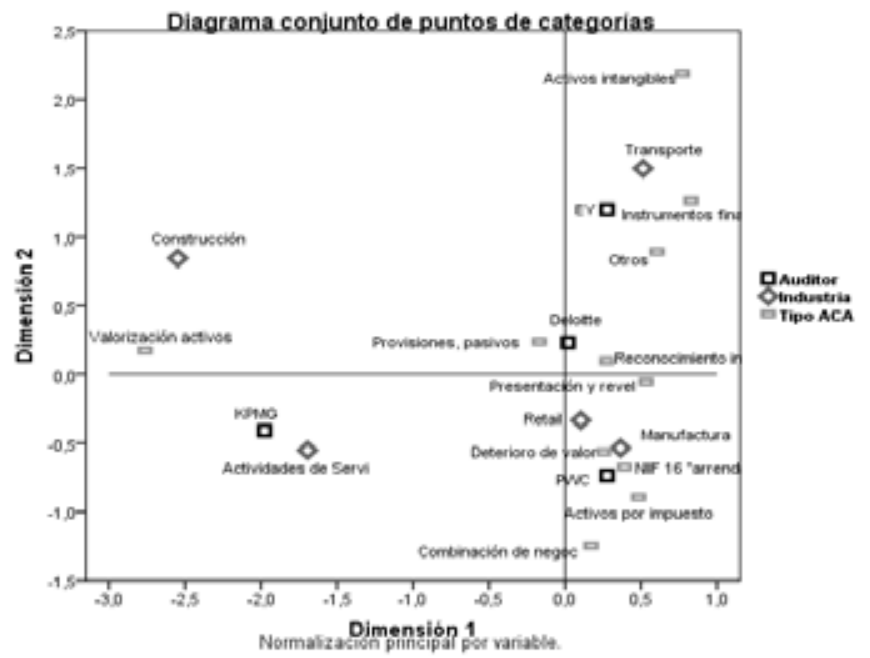

Fuente: Elaboración propia

Este último análisis de correspondencia múltiple resumió las semejanzas y/0 relaciones que se mantuvieron en el segundo año, para luego comparar las relaciones que han marcado diferencia en el análisis. Las principales cualidades que se mantienen en ambos años dicen lo siguiente; La relación entre la industria de construcción en recibir informes del ACA "valorización activos", paralelamente, la relación de auditor KPMG en emitir más ACA "valorización activos", no así industria y auditor. Se observó la semejanza del auditor KPMG, en informar ciertos tipos de ACA que son similares a los que recibe en sus informes la industria de actividades de servicios. Por otra parte, se detectó la relación entre la industria de manufactura en recibir informes de los ACA "combinación de negocios" y "activos por impuestos diferidos, impuestos fiscales y precios de transferencia" por el auditor PWC y finalmente, la relación entre la industria de retail en recibir informes de los ACA "deterioro de valor" y "presentación y revelación de partidas excepcionales y/o no subyacentes".

Al igual que en España, en Reino Unido existen diferencias o cambios generados de 2017 al año 2018 sobre las relaciones entre auditor, industria o ACA, estas dicen los siguiente; Relación en la industria de transporte en recibir el ACA "activos intangibles" por Deloitte el año 2017, cambia una relación solo de la industria en recibir dicho ACA, el 2018 no marca una relación con un auditor específico. Otro aspecto es la relación del auditor Deloitte en informar ACA mayormente a industrias de transporte y retail el año 2017, para el siguiente año Deloitte se ve más relacionado a informar los ACA "reconocimiento ingresos" y "provisiones, pasivos contingentes y otras provisiones contables" sin alguna relación específica de industrias. Continuando, vemos la semejanza en los auditores EY y Deloitte en realizar auditorías en similares industrias y emitir informes con similares tipos de ACA el año 2017, cambia y se separan en el 2018. Una nueva relación en 2018 es 
la de EY en emitir informes con el ACA "instrumentos financieros" $y$ transporte en recibir informes de éste ACA. Además, la nueva relación de EY en informar "otros" ACA, en 2017 esta misma firma se relaciona en informar el ACA "reconocimiento ingresos", que en 2018 no lo hace. La firma PWC presenta sólo relación con el ACA "instrumentos financieros" en 2017, se separa en 2018, y es el auditor EY quien más se relaciona a informar este ACA. Otro aspecto importante de este análisis en Reino Unido indica que en 2017 no se ve una semejanza (0, mayor relación) entre industrias, pero en 2018 se observa la semejanza en las industrias de retail y manufactura en tener similares auditores e informar similares tipos de ACA. En 2018 aparece un nuevo ACA "NIIF 16 arrendamientos efectos o impactos por implementación" que es semejante al ACA "deterioro de valor", informado a similares industrias y por similares auditores. En especial, relacionados con las industrias de retail y manufactura, junto al auditor PWC. También en 2018 aparece el ACA "brexit", que por razones de obtener resultados no sesgados por su baja frecuencia se optó por dejar fuera del ACM, se relaciona directamente en ser informado por el auditor KPMG.

\section{DISCUSIÓN DE LOS RESULTADOS}

El análisis del contenido de los ACA sobre la muestra por conveniencia 20172018 practicado en informes de auditores independientes revela que la tendencia en España con respecto a Reino Unido, en relación a la cantidad y tipos de ACA informados por industrias similares y/o iguales, sitúa a este último conglomerado de países con una práctica de la auditoría mayormente desarrollada, en cuanto al nivel de revelación y detalle de los asuntos técnicos y riesgos evaluados por los auditores durante en el transcurso de su trabajo de campo. Dada la sensibilidad de la información que se revela en estos informes de características "ampliados" puede ser importante, que un error y/u omisión de información tenga efectos negativos no solo para los auditores, si no también para las partes interesadas que consultan permanentemente el juego de los estados financieros y sobre los cuales constantemente se toman decisiones (financieras, operativas, estratégicas, tributarias, etc.)

Al correlacionar los resultados obtenidos en ambos países (España y Reino Unido), puede observarse que los ACA a nivel de las mismas industrias no son coincidentes, es decir, los auditores independientes de las "Big Four" de cada país no consideraron los mismos riesgos en sus auditorías. Aun, siendo las mismas "Big Four" en cada país, no evalúan de la misma forma los ACA, esto puede deberse a las situaciones particulares económicas, políticas, sociales y culturales de cada país. En este sentido, es importante el nivel de experiencia que alcance cada país, a una mayor maduración del mercado mayor será el nivel de revelación de información referentes a los ACA. No cabe duda que este nivel de informes ampliados otorga al mercado mayor confiabilidad y aceptación de información contenida en los estados financieros. 
Dado que las normas de auditoría generalmente aceptadas (NAGA) en Chile están desactualizadas respecto a las normas internacionales de auditoría vigentes en Europa, es importante que los auditores independientes en este país incorporen la NIA (701). Mientras más temprano sea el proceso de adopción, mayor maduración y desarrollo de la práctica alcanzará esta industria permitiendo mostrar a través de sus informes mayor valor agregado a su trabajo que puede ser ampliamente reconocido por los Stakeholders.

Es importante señalar que la NIA (701) pone en evidencia la práctica e involucramiento del juicio y escepticismo profesional del auditor independiente, en cuanto a que deben tomar decisiones importantes a la hora de informar a la Administración y encargados del Gobierno los ACA de mayor riesgo revisados bajo su examen de auditoría.

Conforme a lo observado anteriormente, y en lo referente a lo positivo que puede resultar la implementación de la NIA (701) en Chile, los autores de esta investigación no descartan que también pueden existir efectos negativos; El efecto negativo para los auditores independientes se refleja en el riesgo de exposición en mostrar parte de su trabajo al interés público (el cual podría ser fuertemente cuestionado), y para la entidad auditada el de mostrar información que puede ser utilizada en su contra, como por ejemplo, el uso de ella por parte de sus competidores.

\section{CONCLUSIONES}

La presente investigación analizó el contenido de 95 ACA informados en España y 160 ACA informados en Reino Unido, para los períodos 2017 y 2018 en informes emitidos por auditores independientes en empresas que cotizan en el mercado de valores de estas empresas bajo la NIA (701). El resultado de la presente investigación coincide con las conclusiones obtenidas por los autores (Gambetta, García, Sierra, \& Orta, 2019).

La experiencia en España (ambos años), muestra una tendencia de mayor comunicación de informes en la industria de manufactura, siendo de mayor divulgación el ACA de "Deterioro de Valor" en ambos años y en esta misma industria. Por otra parte en el Reino Unido (ambos años), al igual que en España, denota la tendencia respecto a la cantidad de ACA informados en la industria de manufactura. Respecto a los ACA de mayor divulgación, a diferencia de España, en el Reino Unido fue, "Provisiones, pasivos contingentes y otras provisiones contables" divulgadas en las industrias de Retail (primer lugar) y de Transporte (segundo lugar). Los análisis de correspondencia múltiple para España (ambos años), en términos generales, indica que existe una mayor uniformidad de ACA informados a nivel de industrias, es decir, a nivel revelación se mantienen los mismos ACA en ambos años, sin variación. Del mismo modo, se observa en este país que existe una tendencia que las "Big Four" se mantengan auditando las mismas empresas de un año a otro (baja o nula rotación). 
A diferencia en Reino Unido, el análisis de correspondencia múltiple reveló resultados distintos. Los resultados indican que existe una mayor cantidad de ACA divulgados por industrias y estos son más variados en comparación a España, esto lleva a pensar que existe una mayor maduración y experiencia de la industria de auditoría en Reino Unido en informar asuntos claves de auditoría producto de cambios tempranos en sus normativas de auditorías (a partir del año 2013). Otra situación importante en Reino Unido es la rotación de auditores de empresas de un año a otro, comportamiento que puede estar dado por las fuertes regulaciones que existe en esta industria.

Al adoptarse la NIA (701) en Chile es previsible que la industria de auditoría tenderá a informar los mismos ACA en estructuras de industrias similares y/0 iguales conforme ha sido la experiencia en Europa. Se espera que la decisión de la implementación de estos nuevos informes ampliados se considere lo más pronto posible, de manera iniciar el proceso de discusión y maduración al interior de la industria de auditoría y con sus empresas auditadas.

Esta investigación ayuda a fortalecer una línea de investigación en auditoría que permita efectuar un seguimiento, antes y a posterior de la implementación de la NIA (701) en Chile, por supuesto, también representa una oportunidad para los auditores en otros países que aún no han efectuado cambios en sus informes.

\section{REFERENCIAS}

CARRERA, N., HUMPHREY, C., GÓMEZ, N., \& RUIZ, E. (8 de Mayo de 2007). Mandatory Audit Firm Rotation in Spain: A policy that never applied. Emeral Insight_Accounting, Auditing \& Accountability Journal, 20(5, 2007), 31. doi:10.1108/09513570710779009

CARRERAS, N., GUTIÉRREZ, I., \& CARMONA, S. (abril-junio de 2005). Concentración en el Mercado de Auditoría en España: Análisis Empírico del Período 1990-2000. Revista Española de Financiación y Contabilidad, XXXIV(125), 34. doi:10.1080/02102412.2005.10779552

CHILE, G. D. (agosto de 2017). Ministerio de Economía Fomento y Turismo. Recuperado el 10 de noviembre de 2019, de https://www.economia. gob.cl/estudios-y-encuestas: https://www.economia.gob.cl/wp-content/ uploads/2017/03/Bolet\%C3\%ADn-empresas-en-Chile-ELE4.pdf

CHILE, M. D. (2017). Informe de Resultados: Empresas en Chile, Cuarta Encuesta Longitudinal de Empresas. Santiago: Chile.

COLEGIO DE CONTADOR, D. (2017). Sección AU 700. En C. d. Chile, Normas de Auditoría Generalmente Aceptadas en Chile (Vol. Tomo II, pág. 1468). Santiago, Santiago, Chile: Colegio de Contadores de Chile A.G. 
COLEGIO DE CONTADORES DE CHILE. (23 de agosto de 2017). Colegio de Contadores de Chile. Obtenido de Colegio de Contadores de Chile: https:// www.contach.cl/

CONSEJO DE NORMAS INTERNACIONALES DE AUDITORÍA Y ASEGURAMIENTO. (15 de Enero de 2015). Pronunciamientos finales: Informe sobre los estados financieros auditados - Normas nuevas y revisadas y modificaciones de concordancia. Recuperado el 18 de Noviembre de 2019, de Federación Internacional de Contadores: https:// www.ifac.org/system/files/publications/files/Informes-sobre-estadosfinancieros-auditados-Normas-nuevas-y-revisadas-y-modificaciones-deconcordancia.pdf

CORNEJO, E., \& GUIÑEZ, N. (22 de junio de 2016). Motivaciones de la Gerencia para Manipular la Cifra de Beneficio Contable. Revista Académica \& Negocios, 2(1), 14.

CUESTA, M., \& GARRIDO, P. (2015). El mercado de auditoría en España. Análisis de su estructura y evolución. Valencia, España: Universitat Politécnica de Valencia. Recuperado el 14 de junio de 2020, de http://hdl. handle.net/10251/47156

DELOITTE. (4 de junio de 2013). UKAccountingPlus. (D. UK, Productor) Obtenido de https://www.iasplus.com/en-gb/news/2013/06/frc-isa-700audit-report-revisions

ESPAÑA. (20 de julio de 2015). Agencia Estatal Boletín Oficial del Estado. Obtenido de Gobierno: https://www.boe.es/buscar/act. php?id=BOE-A-2015-8147

ESPAÑA, I. D. (30 de junio de 2019). https://www.icjce.es/. Recuperado el 10 de diciembre de 2019, de https://www.icjce.es/: https://www.icjce.es/ adjuntos/cc-auditoria.pdf

FRC. (1 de junio de 2013). London, England: FRC. Recuperado el 15 de febrero de 2020, de frc.org.uk: https://www.frc.org.uk/getattachment/501de004b616-43c3-8d65-aeaebde19f8d/ISA-700-(UK-and-Ireland)-700(Revised)-Independent-auditors-report-June-2013.pdf

FRC. (1 de marzo de 2015). London, England: FRC. Recuperado el 12 de febrero de 2020, de frc.org.uk: https://www.frc.org.uk/getattachment/561627ccfacb-431b-beda-ead81948604e/Extended-Auditor-Reports-March-2015. pdf

FREEMAN, R. E. (1983). Stockholders and Stakeholders: A New Perspective on Corporate Governace. California Management Review, 15(3), 88. 
GAMBETTA, N., GARCÍA, M., SIERRA, L., \& ORTA, M. (25 de mayo de 2019). Las cuestiones clave de auditoría esperadas en España: ¿son los auditores previsibles? Revista de Contabilidad, 22(1), 9. doi: https://doi. org/10.6018/rc-sar.22.1.354291

HUMPHREY, C., \& MOIZER, P. (15 de abril de 1990). From Techniques to Ideologies: An Alternative Perspective on the audit function. Critical Perspectives on Accounting - ELSEVIER, 217-238. Recuperado el 08 de enero de 2020, de https://www.sciencedirect.com/science/article/ pii/1045235490030217

HUMPHREY, C., LOFT, A., \& WOODS, M. (3 de junio de 2009). The global audit profession and the international financial architecture: Understanding regulatory relationships at a time of financial crisis. Accounting, Organizations and Society - ELSEVIER, 16. Recuperado el 2 de noviembre de 2019, de www.elsevier.com/locate/aos

INTERNATIONAL ACCOUNTING BULLETING. (3 de 1 de 2018). Introduction International Accounting Bulletin | World Survey ... Recuperado el 03 de enero de 2020, de https://accounting.nridigital.com/iab_world_survey_ mar19/market_share_and_fee_data

KAPLAN, R. (Primavera de 1987). Accountants' Liability and Audit Failures: When the Umpire Strikes Out. Journal of Accouting and Public Policy, 1-8. Recuperado el 24 de enero de 2020, de : https://www.sciencedirect.com/ science/article/pii/0278425487900020

LIZASOAIN, L., \& JOARISTI, L. (enero - abril de 2012). Las nuevas teconologías y la ivestigación educativa. El análisis de datos de variables categoriales. Revista Española de Pedagogía(251), 20. Obtenido de https://revistadepedagogia.org/wp-content/uploads/2012/02/251-07.pdf

MONTERREY, J. (octubre-diciembre de 2002). Entre la Contabilidad Creativa y el Delito Contable. Revista Internacional Legis de Contabilidad y Auditoría, 14.

MORALES, F., \& HOLLANDER, R. (septiembre-diciembre de 2009). Contabilidad Creativa en Chile - Una percepción de estudiantes y profesionistas. Contaduría y Administración(229), 20. Recuperado el 15 de enero de 2020

PARLAMENTO EUROPEO. (18 de 12 de 2018). Preguntas parlamentarias. Recuperado el 03 de enero de 2020, de http://www.europarl.europa.eu/ doceo/document/E-8-2018-006354_ES.html

PÉREZ, Y., CAMACHO, M., \& SEGOVIA, M. (enero-junio de 2019). Los Nuevos Informes ampliados de auditoría. Caso: las empresas cotizadas españolas. 
Cuadernos de Contabilidad, 20(49), 18. doi: https://doi.org/10.11144/ Javeriana.cc20-49.ania

ROBALINO, B. (2017). Guía de implementación y aplicación de los nuevos informes de auditoría según NIA 701 en Ecuador. (tesis de pregrado). Guayaquil: Universidad Católica de Santiago de Guayaquil. Recuperado el 15 de Septiembre de 2018, de http://repositorio.ucsg.edu.ec/ handle/3317/8118

RUIZ, E., RODRÍGUEZ , P., \& BIEDMA, E. (14 de octubre de 2015). Barreras de entrada, concetración y competitividad en el mercado de auditorpia español. Revista Española de Financiación y Contabilidad, 45(1, 92-133), 43. doi: 10.1080/02102412.2015.1092232

SILVA, B., \& GARRIDO, C. (2006). La Convergencia a las normas internacionales de información financiera en Chile. Capic Review, 4, 13. 